\title{
Equity in the Driver's Seat
}

A Practice-Driven, Equity-Centered Approach for Setting R\&D Agendas in Education

Babe Liberman and Viki Young, Ph.D.

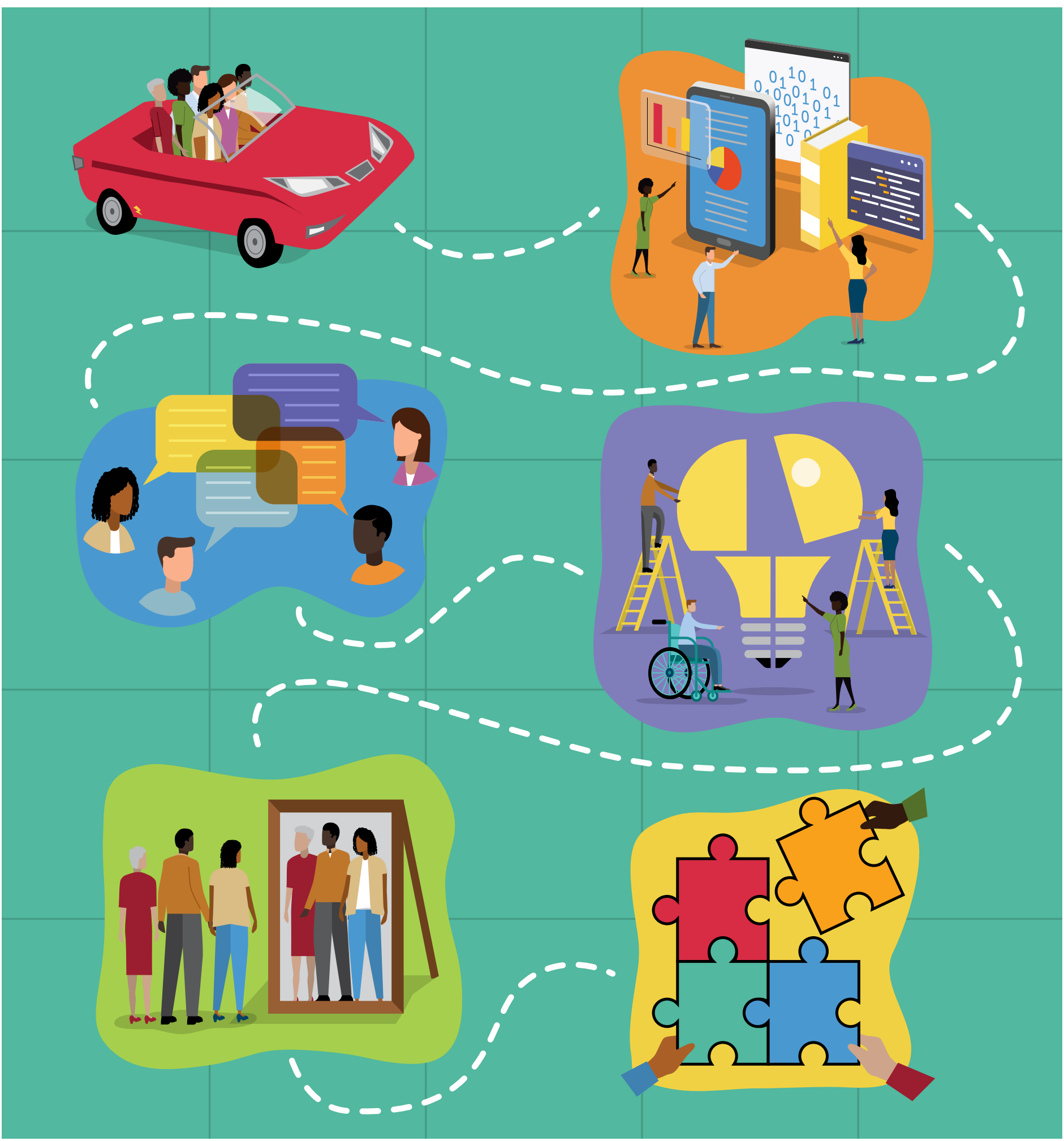

Digital Promise

\footnotetext{
Accelerating Innovation in Education
} 


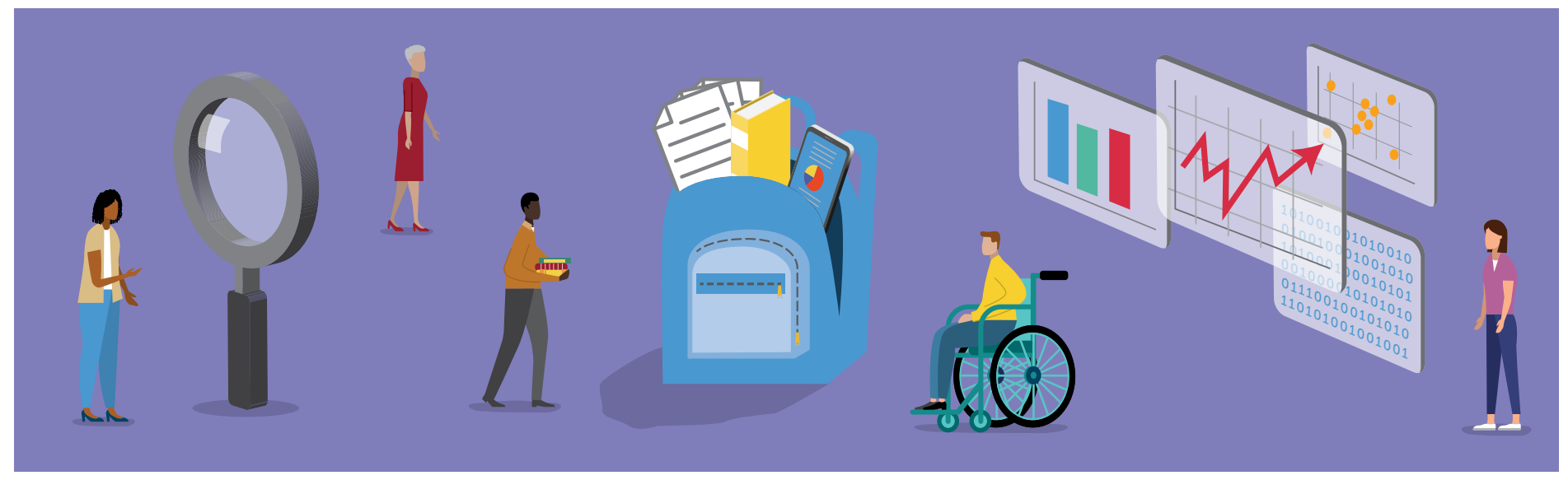

\section{Table of Contents}

Background and Goals

Process

Sessions and Protocols

Sample Agendas

Adolescent Literacy Agenda

Computational Thinking Agenda

R\&D Agenda on Implementation

Learnings

Conclusion

Acknowledgements

Suggested Citation
3

4

9

18

18

22

26

27

31

32

32 


\section{Background and Goals}

Education research often aims to fill gaps in published research or pursue niche interests of researchers, rather than prioritize the challenges schools and districts face on a daily basis. Multiple incentive structures produce this circumstance. For example, funding opportunities prioritize generating new knowledge and early career researchers are rewarded for carving out a distinctive line of inquiry, particularly in academia. More fundamentally, the research establishment accords expert status to those with advanced training and degrees in research methods who've contributed to knowledge-building, so researchers exercise a privileged position to impart knowledge and guidance that is assumed to have generalized value.

In contrast, educators' expertise, often garnered through both advanced education and daily experience, is perceived by society at large as particularized, uncodified, and non-scientific, and is evidenced by the lower status it occupies compared to other professions (e.g., medicine, law, accountancy). Thus, traditionally, those who best understand teaching and learning challenges-educators and school leaders on the ground-do not play a central role in establishing research agendas. As a result, the education studies that researchers design are often not applicable to school districts' most pressing needs.

Even when learner needs are prioritized, research agendas and policy decisions tend to be built around the "average" student, and determined based on group-level statistics. However, assuming that insights about a population automatically apply to all individuals within it is a fallacy; students at the margins-including Black and Latinx students, students experiencing poverty or trauma, students with learning differences, and English learnersoften have very different experiences in schools from their white, middle class, and native English-speaking peers. The needs of students who could benefit most from new innovations rarely drive the design of research and development (R\&D). To prevent this oversight going forward, equity considerations should be front and center in R\&D agendas.

To spur future research that addresses the equity goals of schools and districts, Digital Promise set out to define and test a collaborative process for developing practice-driven, equity-centered R\&D agendas. This process centers on convening a range of education stakeholders to listen to and prioritize the equity-related challenges that on-the-ground staff are facing, while considering prominent gaps in existing research and solutions. We selected two challenge topics around which to pilot this approach and create sample agendas: adolescent literacy and computational thinking. Below we describe this process, share resulting sample agendas, and offer learnings to improve the approach in the future. 


\section{Process}

\section{Selection of Challenge Topics}

The first step in our process was to identify appropriate challenge topics. While we know that schools and districts face a number of pressing challenges, and that some of these challenges are common to educators across the country, for this project we set out to select topics that are relevant and urgent to both the research and practice sides.

\section{We determined the following selection criteria:}

- Strong existing body of field knowledge, with remaining gaps in research

- Hot topic in policy, practice, and equity, with opportunity for marshalling an agenda

- Ongoing need for research synthesis, solution development, and/or technical assistance

Appropriate challenge topics have a research base to start from, but are not fully developed, with meaningful gaps in the research that can be filled based on practice perspectives. These topics should also be timely and responsive to conversations in the field about creating policies that equitably support the full spectrum of students. For this type of persistent challenge, the need to make headway through research synthesis, developing new solutions (e.g., practices, programs, products), and technical assistance is urgent. Based on these considerations, we selected adolescent literacy and computational thinking.

Adolescent literacy meets these criteria because, while we know a lot about how students in early elementary grades learn to read, we know less about how to support adolescents who are severely behind in reading proficiency for their grade level. The equity implications of this challenge are evident in the intersections between reading and writing proficiency and special education designation and English language proficiency. For example, students with dyslexia and students who are not fluent in English often start out at a disadvantage in these subjects, and the gaps can widen by middle and high school. Additionally, teachers at the secondary level often are not trained to teach literacy, various instructional methods used in the primary grades may be less effective with adolescents, and secondary curricula need to have reading materials of interest to adolescents and to be integrated with grade-appropriate subject matter. Moreover, youth who have not been successful in reading and writing through elementary grades can lack confidence and disengage from instructional activities that are inadequately scaffolded for them.

Computational thinking (CT) also meets these criteria because it is an emerging interdisciplinary field that, in many ways, is still being defined. While national economic considerations have consistently buoyed CT as a popular bipartisan issue, marked gaps remain in our understanding of actual best practices for implementing CT competencies across grade levels and in specific subject areas. As a result, we lack clarity regarding how to support novice teachers to effectively engage a diverse range of students in CT learning opportunities. In addition to technical assistance for districts to implement $\mathrm{CT}$, practically 
oriented research syntheses and dissemination strategies are needed to share emergent knowledge with a growing field of interested educators and researchers.

\section{Engaging District Teams}

To center the R\&D agendas for these selected topics on the contextual expertise of teachers and education leaders, we selected four district teams from the League of Innovative Schools. We identified these teams based on their ongoing initiatives related to one of the topics and a demonstrated commitment to equity. Additionally, we were interested in regional diversity among participating teams, and identified districts from four different states across the country. Table 1 lists the participating teams, each consisting of two to three staff members, representing school (e.g., teachers and instructional coaches) and district levels (e.g., directors of instruction and technology $\&$ innovation).

We worked with each district team to articulate a specific equity challenge within their topic area. Districts contributed relevant data and artifacts to illustrate these challenges in context.

Table 1: District Challenges

\begin{tabular}{|l|l|l|l|}
\hline \multicolumn{2}{|l|}{ Adolescent Literacy } & \multicolumn{2}{l|}{ Computational Thinking } \\
\hline $\begin{array}{l}\text { Fox Chapel Area } \\
\text { School District, PA }\end{array}$ & $\begin{array}{l}\text { The district has observed } \\
\text { that students are lacking } \\
\text { sufficient opportunities } \\
\text { to connect with diverse } \\
\text { perspectives in English } \\
\text { Language Arts (ELA) classes } \\
\text { (e.g., through reading a } \\
\text { range of diverse texts), } \\
\text { and teachers are reporting } \\
\text { low empathy skills and } \\
\text { engagement in some } \\
\text { students. }\end{array}$ & $\begin{array}{l}\text { School District, } \\
\text { IA }\end{array}$ & $\begin{array}{l}\text { The district is observing inequitable } \\
\text { course enrollment in high school CT, } \\
\text { computer science, and advanced STEM } \\
\text { electives (in terms of race/ethnicity, } \\
\text { gender, socioeconomic status, and } \\
\text { English Language Learner status). }\end{array}$ \\
\hline $\begin{array}{l}\text { Indian Prairie } \\
\text { School District, IL }\end{array}$ & $\begin{array}{l}\text { The district is working to } \\
\text { design and implement a } \\
\text { more equitable, student- } \\
\text { centered secondary writing } \\
\text { curriculum, with goals } \\
\text { including: developing } \\
\text { students' writing identities, } \\
\text { honoring student voice, } \\
\text { and providing personalized } \\
\text { feedback. }\end{array}$ & $\begin{array}{l}\text { Talladega } \\
\text { Schools, AL }\end{array}$ & $\begin{array}{l}\text { Sche district is looking to effectively } \\
\text { measure and assess student } \\
\text { understanding of and engagement with } \\
\text { core CT competencies, with a specific } \\
\text { focus on two populations: students } \\
\text { from low socioeconomic households } \\
\text { and female students. }\end{array}$ \\
\hline
\end{tabular}




\section{Facilitating Equity in the Driver's Seat Convening}

We planned a full-day convening in San Mateo, California, to bring together the diverse stakeholders key to developing practice-driven, equity-centered R\&D agendas. Organizing and facilitating this convening involved eight key steps, which we illustrate below.

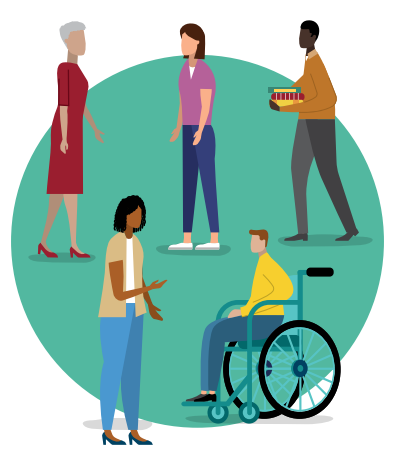

Invite a Range of Experts

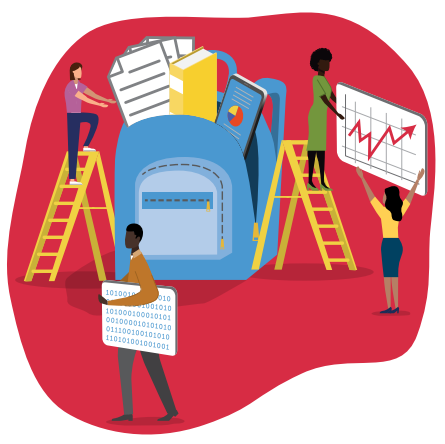

Unpack the Challenge

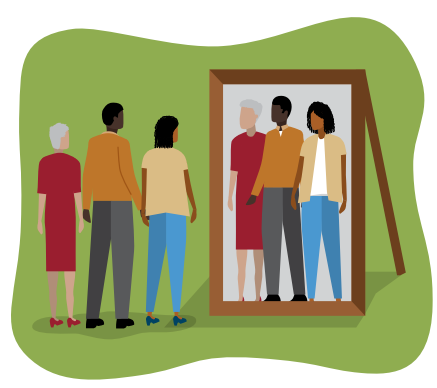

Critically Reflect

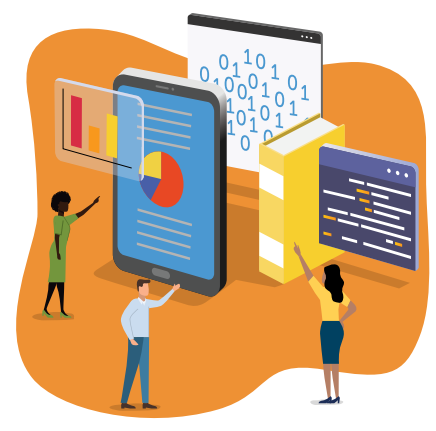

Prepare to Engage

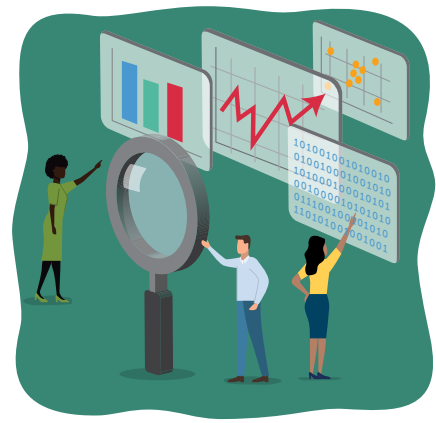

Think with Research

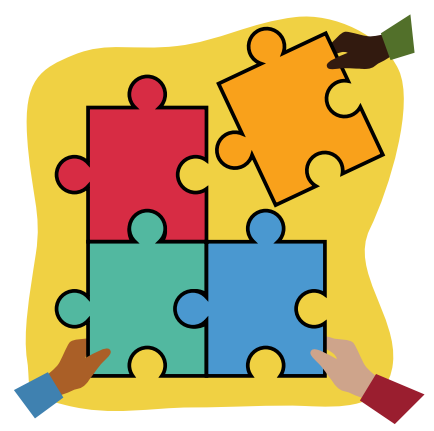

Synthesize Learnings

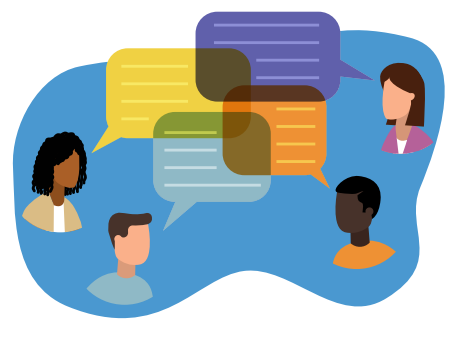

Facilitate Equity Talks

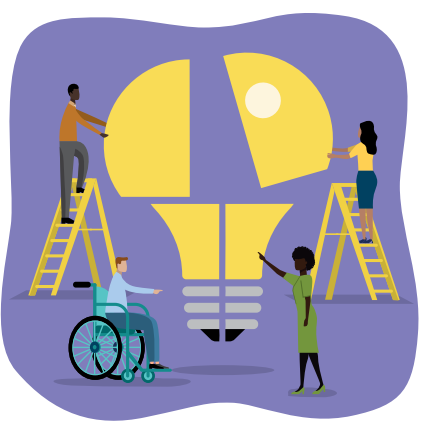

Close the Gaps 
Invite a Range of Experts: Breaking down assumptions about what kinds of expertise count

While research agendas are traditionally developed by select groups of researchers, our goal was to broaden the expertise and perspectives represented, to include more voices in meaningfully shaping the agendas from the start. This purpose informed the convening invitation list. In addition to district teams, we invited a range of research and practice experts with a deep commitment to equity, as well as experience in adolescent literacy and computational thinking. The invitations resulted in 35 attendees, including teachers, coaches, and school and district leaders; developers of educational products and programs; researchers; and funders.

In traditional research agenda-setting processes, research expertise typically takes priority over other kinds of expertise. To prevent researchers assuming or being accorded authority, we highlighted the value of including perspectives from a range of cross-sector roles. We reminded participants that whether they are based in a school, a district, a university, or a nonprofit or community organization, they all have important viewpoints to share. Instead of splitting the group into "practitioners" and "researchers," we invited each participant to offer practice- and research-based perspectives throughout the day, affording all participants with opportunities to question, share, and actively listen. For example, participants with practice expertise could question methods incompatible with classroom realities, and could provide insights into why certain questions should be a priority on the agenda. And participants with research expertise could raise questions based on comparisons and contrasts across multiple contexts, and could suggest promising approaches based on existing research. We hoped that elevating the voices of those who would not typically be involved in setting research agendas would result in more actionable agendas focused on specific and authentic needs in the field. We hope, in engaging in this new process, to broaden the perspectives of all participants and provide valuable new insights for R\&D.

\section{Prepare to Engage: Equipping participants to take part across traditional research and practice boundaries}

To disrupt the traditional dynamic of research knowledge driving R\&D initiatives, and to ensure all participants would enter the convening's discussions armed with working knowledge about the districts' equity challenges and existing field research, we compiled and shared relevant materials including an agenda and pre-reading.

We developed topic overviews to highlight applicable findings in adolescent literacy and computational thinking. Rather than exhaustive literature reviews, these brief overviews provided foundational and recent information on the challenge topics, including high-level introductions and specific findings about current inequities and challenges, strategies for breaking relevant stereotypes, and opportunities for future equity-centered research. We also shared quiding questions that asked participants to reflect on the findings most relevant to instructional practice, the most surprising information, and remaining gaps in knowledge or actionability. 
We also shared artifacts from each district team to illustrate how they experience these challenges in their unique contexts. For example, the Fox Chapel adolescent literacy team provided a diversity analysis of their current middle school book list, as well as a district-level novel selection and vetting form, The lowa City CT team introduced their own district challenge in CT with a hard look at their recent AP Computer Science course enrollment data, including demographic breakdowns. Reviewing research alongside district artifacts that describe authentic equity challenges helped participants recognize that published research is useful, but often does not provide a meaningful starting point for action. Combining rich data from district artifacts with evidence from the literature can result in the nuanced understanding necessary for addressing these challenges in the districts' settings and beyond. 


\section{Sessions and Protocols}

\section{Facilitate Equity Talks: Building trust and gaining inspiration from equity champions}

We kicked off the convening with an equity-related icebreaker and framing talks to build common language around diversity, equity, and inclusion, and to get inspired from dedicated educators who champion equity in their work with students. Because many participants didn't know each other and we only had one day together, we knew we needed to help build trust and openness straight away. Each participant selected from the following equity sentence stems and introduced themselves to the full group in 30 seconds or less. It was important that everyone share, in their own words, what brought them to the convening, to underscore the shared passion and commitment to students.

- I'm an education equity superstar because

- My education equity challenge is

- I'm passionate about education equity because

Next, we invited two equity champion practitioners to deliver keynote presentations to inspire participants with exemplars of equity-centered instruction in both of the topics. Jessica Stovall, doctoral candidate and teaching fellow at Stanford, drew upon her 11 years as a high school English teacher

\section{"We are all in the same place, though our environments look a little different. We all want what's best for kids and teachers." - District-Level Leader} working to interrupt systemic racial disparities in achievement to discuss the harm in "teaching for equality." She shared her experience adopting an equity framework, including peace circles, in her classroom, and how she explicitly taught students that things can only be fair if everyone begins at the same starting point. Kennan Scott, an experienced STEM educator who started his career as an engineer, shared his vision for "making computer science and CT relevant in the hood." To articulate the challenge, he combined statistics on the lack of diversity in tech fields with firsthand experience of the isolation and obstacles faced by people of color working in STEM fields. He presented ideas for boosting student agency through meaningful STEM learning. The powerful stories from these presenters and real-life examples of advancing equity in their settings were inspiring to convening participants. 
We then posed some reflection questions for participants to consider throughout the day. At the heart of this convening was an effort to demonstrate what a practice-driven, equity-centered R\&D agenda looks like and by doing so, to derive a ground-up understanding of what distinguishes such agendas from those that result from more traditional processes. We wanted to learn from participants' experiences whether and how those distinctions arose, and thus asked participants to reflect metacognitively on the following questions, which we returned to at the end of the day:

- How is this type of R\&D agenda similar to traditional research agendas?

- How is this type of R\&D agenda different from traditional research agendas?

-What indicators demonstrate that the agenda is truly practice-driven?

- What indicators demonstrate that the agenda is truly equity-centered?

For the next several sessions, we split the group into two tracks; one for each challenge topic. Each track of 16-20 people featured two district teams as well as Digital Promise facilitators and external researchers, non-profit professionals, funders, and teachers and education leaders. 


\section{Unpack the Challenge: Centering discussion on districts' specific equity challenges}

We intentionally structured the day to begin with the specific equity challenges that the invited district teams were facing, rather than starting from a research perspective. Our hunch was that starting with the districts' challenges would result in research questions, development priorities, and action plans that would be relevant and usable in practice. To unpack each challenge, we facilitated a structured consultancy protocol. Non-presenting participants adopted the role of "consultants" who listened with two purposes: 1) how they could help district teams further clarify and articulate their equity challenges, and 2) as consultants, what their own ideas were for tackling these challenges (including through future R\&D).

Table 2: Equity in the Driver's Seat Consultancy Protocol

\begin{tabular}{|l|l|}
\hline 1. District team presents equity challenge & $\sim 10$ minutes \\
\hline $\begin{array}{l}\text { 2. Consultants ask clarifying questions; } \\
\text { district team answers }\end{array}$ & $3-5$ minutes \\
\hline $\begin{array}{l}\text { 3. Consultants ask probing questions; district } \\
\text { team answers }\end{array}$ & $\sim 10$ minutes \\
\hline $\begin{array}{l}\text { 4. Consultants discuss equity challenge; } \\
\text { district team listens (small groups) }\end{array}$ & $\sim 15$ minutes \\
\hline $\begin{array}{l}\text { 5. District team reflects and shares new } \\
\text { thinking }\end{array}$ & $3-5$ minutes \\
\hline \begin{tabular}{l} 
(Repeat with second district team) \\
\hline
\end{tabular}
\end{tabular}

Among both the adolescent literacy and computational thinking groups, one school district team described their selected challenge for the consultants, sharing information about the groups most impacted by the challenge and any emerging strategies being tested. Next, to ensure consultants had enough context to provide useful feedback, consultants asked the district team clarifying questions, or simple questions of fact. Once the group was clear on those details, consultants transitioned into probing questions: deeper, open-ended questions that empowered the district teams to reflect on and think about collaboratively addressing their problem. These questions were meant to promote inquiry, challenge assumptions, or create a paradigm shift for district teams. 


\section{Sample Probing Questions}

- What barriers are keeping you from fully tackling this challenge?

- What are the assets that can be tapped to fully tackle the challenge?

- Why do you think is the case?

- What do you assume to be true about ?

- What was your intention when __ ?

- What's another way you might __ ?

- What is the best thing that could happen?

- What are you most afraid will happen?

Next, we broke into smaller groups to invite more voices to contribute. Consultants in each small group discussed the equity challenge the district presented, worked to define the issues more thoroughly and objectively, and provided suggestions about actions for the district team to consider taking. Finally, district team members had a chance to share comments that resonated during the consultancy, as well as any new insights or perspectives. We repeated the process with the second district team.

Common trends emerged as well as contextual observations about what was unique to each district, including the local policy context and community assets, opportunities, and barriers, all of which are key real-world considerations that can impact R\&D. Researchers appreciated the opportunity to consider these elements before jumping into ideas for filling gaps in the research.

The consultancy protocol was thought-provoking for convening participants; one district leader shared plans to use a consultancy protocol in her district meetings. In the future, we'd allow even more time for exploring and interrogating specific district challenges, and would consider suggesting probes to keep the group centered on the equity aspects of these driving questions.

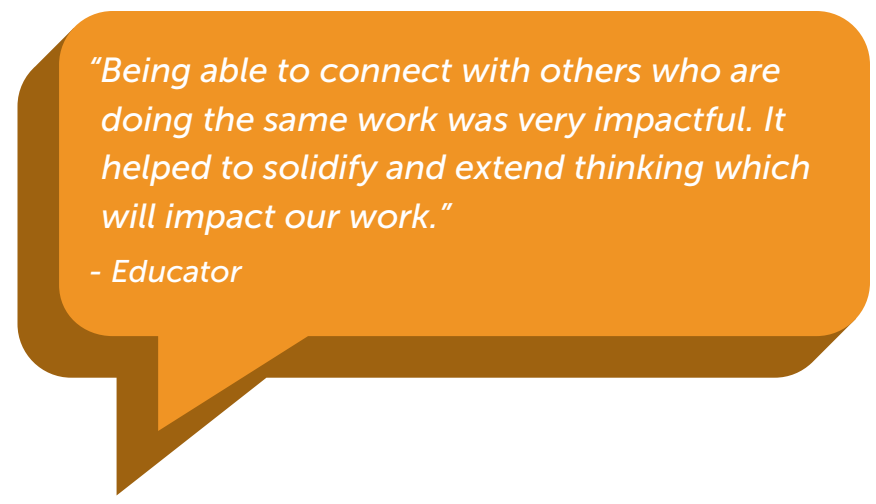


The goal for the next session was to discuss how existing knowledge from the research literature could help address the district teams' specific challenges. Instead of starting from the perspective of key findings in the literature, this discussion was centered on the research most relevant for these communities. While researchers typically kick off this type of dialogue, we flipped the script to start the discussion from the district teams' perspectives. Each district team shared their viewpoints on the most interesting and most useful information they learned from the topic overviews. They also described the ways the research overview informed their thinking about their specific context and challenge. Finally, district teams shared what else they would need to know to move forward in tackling their challenge.

The group then responded to and built on the district team's ideas. Participants with a formal research background had the opportunity to share additional relevant research findings, including particular studies and ongoing initiatives addressing some of the adolescent literacy or CT issues being discussed. Facilitators supported the group in probing research ideas, with attention to usefulness for practitioners and focus on equity. Participants captured themes about knowledge gaps on Post-it notes, to be used in the next round.

While the topic overviews we provided were content-driven and focused on what we know from research about adolescent literacy and CT, creating equitable change in schools is complex and broader than these subject matter-specific challenges. In this session, districts identified wanting support in different aspects of change management, including strategies for gaining school board support, engaging teacher leaders as advocates for specific changes, and scaling an innovation or initiative beyond the original group who worked on it. They also wanted to learn about relevant instructional strategies for diverse learners and ways to support historically marginalized students. Providing topic overviews on broader subjects like school reform processes (including sustainability and scale), learner variability, deeper learning, and culturally responsive teaching could have benefited participants in this session. For example, learning from thoughtful resources like Cultivating Genius: An Equity Model for Culturally and Historically Responsive Literacy and Designing for Rightful Presence in STEM: The Role of Making Present Practices could have provided a useful framework for thinking about how historically marginalized students experience school and specific subjects. 


\section{Close the Gaps: Building on the group's varied expertise to propose R\&D priorities}

To coalesce the group's understanding about gaps in knowledge and to begin collaboratively defining an agenda for future $R \& D$, this session involved drawing from both the experience of district teams and the experience of others in the room who have worked on the topic in a range of settings. Facilitators introduced two categories for setting the agenda: 1) What else do we need to know (research questions), and 2) what solutions do we need to support districts in using the research (development priorities)? Table 3 shows questions participants considered in their attempts to surface key R\&D areas.

Table 3: R\&D Agenda Setting Questions

\begin{tabular}{|c|c|}
\hline Research & Development \\
\hline $\begin{array}{l}\text { What else do we need to know? What do you } \\
\text { feel is missing from the research based on } \\
\text { practice-relevant challenges? }\end{array}$ & $\begin{array}{l}\text { What would make this research more usable } \\
\text { for districts? What would districts need to take } \\
\text { advantage of this research? }\end{array}$ \\
\hline $\begin{array}{l}\text { - What are some topics/areas about which } \\
\text { we don't know anything yet (e.g., effective } \\
\text { strategies for engaging students experiencing } \\
\text { homelessness in writing)? }\end{array}$ & $\begin{array}{l}\text { - Is there a need for accessible communication/ } \\
\text { synthesis? }\end{array}$ \\
\hline $\begin{array}{l}\text { What is the practical impact of proposed } \\
\text { research questions? What would practitioners } \\
\text { do with the answers? }\end{array}$ & - Is there a need for training/capacity building? \\
\hline $\begin{array}{l}\text { - How can you frame this as a question of } \\
\text { equity? }\end{array}$ & $\begin{array}{l}\text { - Is there a need for equity support to ensure } \\
\text { that research findings are relevant to specific } \\
\text { contexts/subgroups (e.g., students, families, } \\
\text { educators of color, those experiencing } \\
\text { homelessness, non-native English speakers)? }\end{array}$ \\
\hline
\end{tabular}

To encourage a broad array of perspectives, participants individually brainstormed ideas for both categories using Post-it notes, then organized and clustered similar ideas within each category. Participants took a gallery walk to review the ideas, then split into two or three smaller discussion groups based on shared interests, with at least one group focused on a research-related topic and at least one other on a development-related topic. For example, the CT participants split into two small groups-one on researching how factors in CT instruction affect diverse students' self-efficacy, experience, and desire to persist in computing, and another on developing new CT assessment tools and resources. Facilitators prompted participants to build on their ideas by keeping equity considerations (e.g., whose issues are prioritized in R\&D, who is included in R\&D processes, and how are R\&D decisions made) front and center, and ensuring the R\&D ideas are usable for the field. 
Facilitators were transparent that this session, like the larger convening, was an experiment and a learning experience for everyone involved. Notes from the small groups showed that the resulting ideas were still complex and messy at the close of these discussions. This complexity was expected; co-design processes are cyclical. More work to organize and synthesize ideas after the convening and additional rounds of feedback would be required. Nonetheless, the collaborative and generative brainstorm process was engaging for participants and ultimately informed a more polished R\&D agenda.

Finally, participants had the opportunity to participate in an "un-conference," in which they worked in small groups to move an idea from the R\&D agenda discussion forward. This activity was the most action-oriented of the day, and invited participants to think about concrete next steps for their respective contexts including stakeholders who should be involved, timelines, and metrics for measuring success. Most district teams elected to work together. They were joined by thought partners whose role was to repeat back what teams described as their goals and steps, ask clarifying questions to ensure the group was on the same page, probing on the actionability and equity focus of proposed steps, and offer resources and practical suggestions based on their own experiences.

"It gave me a better understanding of where

we are heading as a district. This helps me

identify my piece of the puzzle and what I

can do to maximize my impact."

- District-Level Staff Member 


\section{Critically Reflect: Considering practice-driven and equity-centered indicators}

Reflection involves actively exploring an experience to gain new or greater understanding. Reflection was critical in this process because we were experimenting with a new process to flip the traditional approach to setting education research agendas on its head. It was important to unpack the experience to determine if and how it differed from the customary approach, and to reveal which elements were effective and which to improve or replace. Previewing the reflection questions at the beginning of the convening primed participants to be reflective partners throughout this learning experiment.

Building on their new insights from the day's sessions, including equity talks, consultancy protocols, and planning to fill the gaps, participants revisited the reflection questions. They posted new and updated ideas, which they reviewed alongside the morning's Post-it notes and responses from others. Participants reflected on the development of their thinking, and described what they might do differently in their roles as researchers, educators, policymakers, and funders to achieve equity goals, based on what they learned. In this round, their responses were more nuanced and grounded in their shared experience of digging into district challenges to spark ideas for R\&D. Common themes included the importance of personalized and contextualized research questions; content that is practical, relevant, and actionable; and having an explicit focus on the needs of historically marginalized groups.

With the last reflection question, participants discussed how the process itself could be improved. Key ideas shared included providing additional equity framing, spending more time articulating district challenges from the standpoint of a practical timeline for reform and improvement, and introducing more scaffolding to the discussion about the existing literature on adolescent literacy and computational thinking. To close the day and offer the opportunity for further critical reflection, participants completed a short feedback survey. Ideas that participants shared via the sessions and the survey were incorporated into the draft agendas.

"This experience has been truly illuminating and practicechanging at such a deep level. It's as if you and it are reshaping me...I'm still nascent in this, but I catch myself (sometimes) pausing before entering into solution/ resolution mode, and try to ask and ask and ask and discover and discover. To the creators, leaders, speakers, funders AND participants - thank you!!"

- District-Level Leader 


\section{Synthesize Learnings: Integrating ideas into relevant and actionable R\&D agendas}

Through this process, our goal was for the resulting agendas to reflect the nuance and diverse perspectives that took part in the discussions. We knew we would likely come out of the convening with scattered notes, structured in divergent ways, but it was critical to see what we could learn from this flipped approach. After the convening, we reviewed session notes, notes from Digital Promise internal reflection sessions, and participant feedback survey responses and synthesized them into themes. For each topic we noted challenges and organized research questions and development priorities into the following categories: Student Learning/Outcomes, Assessment/Measurement, Professional Development, Implementation/Scaling, and Policy/Political Context.

We compiled the following draft R\&D agendas, based directly on these themes, in partnership with district team members and other convening participants. 


\section{Sample Agendas}

We present illustrative examples of R\&D agendas based on the driving questions that district partners raised during the convening. The organization of each strand's sessions into a structured R\&D agenda required us to interpret the discussions and rearrange the themes into a logic that stands on its own in this description of the agendas.

These are not intended to be comprehensive research and development agendas that build on a thorough literature review and do not purport to speak for or direct the overall field.

Rather, these R\&D agendas go deeper into the specifics underpinning the districts' driving questions to illuminate the specificity of practitioners' questions and the inherent difficulties for research and evaluation to answer narrow questions in a timely way. They also address the comfort we need to have to extrapolate from existing data to inform decisions, not determine decisions, and the gap between knowledge and usable knowledge.

\section{Adolescent Literacy Agenda}

\section{Adolescent Reading}

\section{Driving Questions Arising from District Challenges}

- How do diverse texts change student experiences in reading?

- What are the implications for teachers using diverse texts in middle school ELA classes?

\section{What research in adolescent reading instruction can be applied directly to instructional program decisions as they relate to the driving questions?}

- What student outcomes are related to students reading more diverse texts in the curriculum?

- What are the target skills for students to learn from using diverse texts?

- What do students learn from using diverse texts that differs from using traditional texts?

- Does reading diverse texts improve student empathy for those who are othered or marginalized?

- What are appropriate measures of empathy?

- How do we know if diverse texts improve students' empathy for the Other?

- Does reading diverse texts improve student engagement in reading?

- How is student engagement affected when the protagonists, settings, and issues are embedded in cultures they have little knowledge of?

- What equity understandings and empathy skills do students need to develop to approach texts with different cultural meanings?

- How do students' selections for personal reading change as a result of exposure to diverse texts?

- Does reading diverse texts improve reading proficiency?

- What is the theory of change/path? Through increased engagement and practice? 
- How does reading diverse texts show up in student writing?

- Topic selection?

- Word choice?

- Connections between text and self?

-What knowledge and skills do teachers need to meaningfully teach with diverse texts?

- What equity challenges arise when teachers from dominant white culture teach students from dominant white culture about characters and issues embedded in literature from different cultural contexts?

- What equity and empathy knowledge and skills do teachers need?

What tools and resources do educators need to leverage and apply knowledge gained through research pertaining to the driving questions?

- Teaching guides, tools, and resources that support middle school teachers in helping students make connections beyond their own culture

- Scaffolding for students to learn about worlds other than their own without reinforcing stereotypes

- Culturally responsive learning environments that include diverse texts in the curriculum

- Culturally responsive teaching strategies for middle school ELA

- Culturally responsive practices that school leaders, coaches, curriculum directors can look for in ELA instruction

- Culturally responsive practices that families and communities can adopt

- Resources and supports for teachers to teach empathy

- Sample prompts and discussion models of readers approaching texts with empathy

- Reflection questions, vocabulary, and other indicators of what empathy sounds like when students discuss texts

\section{Adolescent Writing}

\section{Driving Questions Arising from District Challenges}

- How do students develop their identity as writers and improve as writers?

-What supports do teachers need to help their students develop as writers?

What research in adolescent writing instruction can be applied directly to instructional program decisions as they relate to the driving questions?

\section{Students as Writers}

- Does student-centered writing instruction develop student identity as writers? If so, in what ways?

-What outcomes are related to student-centered writing instruction?
- Practice habits?
- Engagement? 
- Identity?

- Proficiency?

- Agency?

- What instructional strategies promote student writing identity?

- How do they differ for different types of students (e.g., students who have not been successful writers in the past, EL students, students from diverse economic and sociocultural backgrounds)?

- How is writing identity related to writing proficiency?

- What are useful measures of writing identity?

- What strategies improve students' engagement in writing?

- Does student-centered writing instruction improve students' engagement in writing? If so, in what ways?

- Does collaborative student writing improve student engagement in writing or other outcomes? If so, in what ways?

- What strategies improve equity in writing outcomes?

- How do text resources used to build argumentative writing skills affect equity in outcomes, given students' differing background knowledge related to writing prompt topics?

- How does student choice scaffold student learning in argumentative writing?

- Is student voice in writing related to more equitable writing outcomes?

- In what ways do mentor texts (exemplars) help students learn to write?

- Do they motivate students? Demotivate students?

- Do they undermine students' understanding of the need for revisions?

- Is diversity in mentors texts important for student engagement and learning? If so, in what ways?

- What are measures of writing proficiency that are meaningful and manageable for teachers to collect?

\section{Teachers as Writers and Writing Instructors}

- How do teachers develop self-efficacy and identity as writers?

- Do teachers' self-efficacy and identity as writers lead to better writing instruction?

- What is the relationship between teachers' self-efficacy as a writer and developing students' identity as writers?

- Does teachers' developing a writing identity support students' developing writing identity? If so, in what ways?

- How do teachers' developing identity as writers relate to their understanding of students' struggles in writing?

- Do teachers' understanding of developing a writing identity influence their practice to provide equitable access to writing instruction? 


\section{What tools and resources do educators need to leverage and apply knowledge gained through research pertaining to the driving questions?}

\section{Student Supports}

- Instructional strategies to promote student understanding of strong writing and a balanced approach to teaching grammar and conventions as well as other style and analytic elements of writing

- Strategies, tools, and resources to help teachers build writing identity among adolescents

- Differentiated strategies for building writing identity among those who have been less successful in writing in the past and those with proficient writing skills, for those lacking confidence in writing

- Practice indicators of culturally responsive writing instruction for school leaders, coaches, curriculum directors look for in the classroom

\section{Teacher Supports}

- Sustained professional learning strategies to develop or refine teachers' foundational writing instruction skills

- Differentiated professional learning opportunities for novice and experienced writing teachers

- Strategies for teachers to model (for students) vulnerability in writing, e.g., thinking/ prewriting, revision, so students understand that writing is a process, iterative, and requires practice

- Professional learning for teachers focused on providing effective feedback on student writing

- How does a common writing rubric change teachers' writing instruction practices, if at all?

- Professional development strategies to support writing across the curriculum

- Differentiated professional learning for non-humanities teachers

- Curriculum and structural changes to facilitate more writing instruction and to build writing into non-ELA courses 


\section{Computational Thinking Agenda \\ Access to CT Opportunities}

\section{Driving Questions Arising from District Challenges}

- How can schools increase student enrollment of underrepresented groups (e.g., girls, Black/Latinx students, and English learners) in high school elective computer science (CS) courses and other courses that integrate computational thinking (CT) competencies?

- What are the implications of integrating CT across subject areas in grades $\mathrm{K}-8$ (providing exposure to all students) for student learning and persistence? How does assessment work when CT is integrated into other subjects? Can gains in CT be effectively measured apart from the particular subject (i.e., math, science, ELA) in which it is being incorporated?

- Beyond equitable exposure to CS courses and CT integration, how can school systems embed equity considerations into the design of curricula, pedagogies, and professional learning for CS courses and CT integration?

\section{What research in $\mathrm{CT}$ teaching and learning can be applied directly to instruc- tional program decisions as they relate to the driving questions?}

- How do factors in CT instruction (e.g., teacher, peers, course materials) affect diverse students' self-efficacy, experience, and desire to persist in computing?

- How does student identity factor in students' decision to enroll and persist in a computing course? Is there content in introductory courses that consistently appears more relevant to certain populations (e.g., girls, Black students), leading to greater persistence in advanced STEM and CS courses?

- What is the effect of specific types of teachers (e.g., teachers of color, female teachers) on students pursuing future CT opportunities (e.g., course enrollment, extracurriculars)?

- How can teachers promote a sense of belonging within CT learning opportunities in students underrepresented in CS/STEM?

- Which barriers to participation in CT are universal across underrepresented groups, and which barriers are specific to sub-groups?

- What messages are effective or motivating to support underrepresented students to enroll or persist in CT-related electives?

- How can teacher pedagogy and professional learning promote equitable CT opportunities?

- What language and strategies do effective CT teachers use?

- What does culturally relevant CT pedagogy look like?

- How can CT content and skills be integrated into existing teacher PD?

- How can CT-specific PD be enacted in classroom practice and effectively build upon itself?

- How can schools of education successfully prepare more teachers with knowledge and skills to integrate CT across subjects? How can schools of education prepare more teachers of color to teach CT content and skills? 
- What are the effects of CT integration at the student, school, and district levels?

- Longitudinally, where is the research suggesting that universal access to CT integration or exposure in elementary and middle school leads to broader participation in high school elective CS coursework?

- What are the effects of inequitable elementary access to CT integration (e.g., some elementary classrooms have trained teachers and effective explicit CT focus and others have almost no exposure) on student learning and persistence in STEM?

- Does CT integration into core subjects enhance disciplinary learning (e.g., improve outcomes in math or science)?

- How can CT be effectively implemented at the system level?

- How can schools build more interdisciplinary CT connections that emphasize computing as a tool for communication and creativity (and not simply for CS)?

- Who defines CT for a district and/or school and how does such a definition affect implementation across grade levels (i.e., integrated courses versus stand alone courses)?

- What is the role of vocabulary in promoting a unified vision for CT education?

- How do state standards in CS support districtwide CT integration? To what degree are districts enacting such standards and what is their impetus to do so?

- How are states and districts positioning CT integration within the curriculum (i.e., career and tech education versus STEM)?

- How do CT integration opportunities in K-8 affect high school course offerings, student enrollment, and teacher demographics?

\section{What tools and resources do educators need to leverage and apply knowledge gained through research?}

- Resources for diversifying CT enrollment

- Recruitment strategies to bring underrepresented groups (e.g., students of color, girls, English learners) into CT opportunities and tap into student interests

- Algorithm for student scheduling or other tools to support proportional course enrollment

- CT professional development resources

- Professional development offerings for CT educators to learn about and gain skills around promoting diversity, equity, and inclusion

- Teacher PD on specific CT competencies and developing teacher understanding of where/how CT fits into the curricula of different subject areas

- Teacher PD on conducting action research to address the CT and equity challenges coming up in classrooms

- Resources to help teachers understand how they are already employing elements of CT so they can refine their craft with explicit exploration of CT

- Tools/talking points for building teacher buy-in around CT integration

- CT curriculum resources 
- District curriculum policies that make room for CT integration

- Lesson plan examples that showcase CT integration (at elementary, middle, and high school levels, across a range of core subjects)

- CT teacher recruitment resources

- Strategies for recruiting more teachers of color and female teachers to teach CS courses and CT integration

\section{CT Assessment}

\section{Driving Questions Arising from District Challenges}

- How can we assess students' understanding of and engagement with CT?

- How can we assess the equity of CT programs to ensure specific needs of students from marginalized backgrounds are being met?

- How can we increase awareness in students, families, and community members of CS as a course of study, CT as core competencies, and their value as a career pathway?

\section{What research in CT assessment can be applied directly to instructional pro- gram decisions as they relate to the driving questions?}

- What elements of student understanding of and engagement with CT can and should be measured?

- How can teachers assess student conceptual gains in CT?

- What does "success" in CT coursework look like? To what degree does success in CT coursework and activities correlate to academic success in other disciplines?

- Does CT promote student engagement? How can educators use formative assessment to increase student engagement in CT courses?

- Does CT promote problem-solving more broadly? How can we measure problem solving ability?

- Does CT promote communication and creativity more broadly? How can we measure these skills?

- What equity measures do we need to assess in order to support students from marginalized backgrounds in CS/CT courses?

- Are the assessments currently in place culturally biased?

- How can we measure the impact of culturally responsive CT pedagogy/curriculum?

- How can teachers implement data collection methods that take students' marginalized experiences in account?

- How can diverse students bring their identities into the classroom to shape CS courses and CT integration coursework? How can student self-assessment support equity in CS courses and CT integration coursework?

- How can we assess district capacity to develop equitable computing K-12 pathways (or provide a tool to gauge developmental trajectory toward equitable pathways)?

- How can we build a shared community understanding about the power of CT? 
- How can the definitions and language around CT competencies be communicated accessibly, both for a general audience and specifically for community members from historically marginalized groups?

- How can we measure changes in perspectives (of students, teachers, families, and community members) on computing as a discipline and potential career pathway?

\section{What tools and resources do educators need to leverage and apply knowledge gained through research?}

- CT assessment tools and resources

- Compilation/framework of CT assessment tools that already exist, sortable by CT competency, subject area connections, and effectiveness

- A compilation of surveys and reflection/self-assessments to understand how students experience and understand CT and measure student opportunities to engage with CT competencies

- Rubrics, checklists, and sample student work to demonstrate what quality CT learning looks like for different grade levels and subject areas

- Data collection instruments that reflect the unique challenges and opportunities that exist in a specific district context

- Formative assessment tools to see if/how CT programs are improving student learning outcomes in other disciplines as well as wider problem solving/student initiative

- "Novel problem" tool to understand how students solve a problem and articulate their process

- CT communications resources

- User profiles to show how different subgroups relate to or engage with CT topics

- Talking points/resources to get parents and community members excited about the potential of CT (including connections to the future of work)

- Talking points to shift the narrative around assessment (move beyond math and reading scores)

- Language/tools to help communities understand how programs that target specific subgroups could be broadly beneficial

- Clear definitions of each CT competency and age-appropriate language to use for each CT competency (e.g., K-2, 3-5, 6-12) 


\section{R\&D Agenda on Implementation}

Ultimately, the R\&D agendas as illustrated here have the goal of supporting teachers in improving literacy, writing, and CT instruction, especially for the most underserved students. As with any educational change effort, a series of implementation and scaling questions arose in the discussions and formed an integral part of an R\&D agenda. Below, we list some questions for consideration. Although they are written as generally applicable, we argue that the questions regarding inclusion and equity need more emphasis-by researchers, developers, funders, and policymakers-in understanding educational improvement of all kinds.

\section{Implementation and Change Strategy}

-What strategies support creating change at local levels (e.g., local champions, stakeholders, involvement and buy-in at multiple levels)?

- What progress indicators specific to a particular intervention support meaningful midcourse corrections?

- What process allows for efficient, low burden, and meaningful data collection on progress indicators and outcomes throughout implementation?

\section{Inclusion and Equity in Implementation, Sustainability, and Scale-Up}

- How are teachers' voices used in creating change (e.g., teachers as readers and how they make their reading choices, or teachers using computing to address community challenges)?

- How are teachers supported and taking ownership to enact change in their own classrooms, schools, and districts (e.g., connecting past to current literature)?

- How are minority and marginalized community voices included and heard in the change process? How do their voices moderate the implementation and improvement process?

- How are political considerations factored into improvement design and implementation decisions? How do political considerations intersect with equity considerations (e.g., commitment to the traditional canon in middle school literature and reflecting diversity in contemporary society)?

- How do implementation barriers and facilitating factors vary for different student populations?

-What kinds of outcomes matter as measures of equity? Are equity outcomes valued at least as much as efficiency outcomes in understanding sustainability factors? How are those equity outcomes measured?

- How do scaling strategies adapt innovation and implementation to the lived experiences of marginalized communities in new contexts or locales? 


\section{Learnings}

In reflecting on the process and resulting R\&D agendas, we began by noting some similarities and contrasts with R\&D agendas that might arise from more traditional processes. The two approaches share similar purposes, including a focus on building a body of knowledge; a commitment to identifying practices that work for teachers and students; articulation of research questions relevant and responsive to school and instructional environments; and a desire for practitioners to be able to use research resulting from agenda to substantiate their decisions.

Beyond these overlapping purposes, we also observed whether and in what ways the sample adolescent literacy and computational thinking R\&D agendas evidence elements that distinguish them from R\&D agendas produced through more traditional methods. It was relatively easy to identify explicit signs of being practice-driven.

- The starting point for identifying R\&D questions begins with the practical field (e.g., district leaders, school leaders, educators, community members), who drive the conversation to frame the agenda. Practitioners are involved from the start to codevelop the agenda rather than giving input or advice in response to a researcherdefined process or serving only as research sites or subjects on programs defined first by research literature and external expertise.

- Some of the research questions shaped by the challenges districts presented were very specific and context-based. Thus, the sample agendas are just that: convening participants generated an agenda, not the agenda. These agendas are not intended to define comprehensive and long-term research programs around which to marshal the field. Replicating this convening with different districts and different articulated problems in adolescent literacy or computational thinking would likely yield a different overall agenda, even if certain core questions overlapped. An important validity check might be a subsequent phase of workshopping draft agendas with colleagues from the same districts to see if the R\&D questions resonate with them.

- The specificity of some questions also means that research exactly matching the question often doesn't exist, and is unlikely to unless the district undertakes that research. Existing research can still be helpful, though using it would require comfort with and facility in extrapolating from various study findings and mediating those findings with one's own knowledge of the local contexts and goals. Additionally, we could enhance our facilitation with an iterative process that collaboratively moves the discussion from the specific, practice-based challenges and partially formed hypotheses to articulate questions answerable with research methods.

- Practice-generated questions tend to be multidisciplinary. Addressing these questions requires familiarity with and capacity to build on or triangulate across multiple bodies of research literature. Again, practitioners' understanding of their own contexts would mediate how they integrate and apply findings across disciplines. 
- Some needs that participants identified underscored a desire for a workable solution to try. That is, their motivation for consulting the research or other available resources lay in developing or codifying strategies or interventions to address the specific problems of practice they raised. Relevant research can inform directly or tangentially to support practitioners in forming a reasonable approach to the problem they determined, as guided by their instructional expertise and knowledge of their students. It may be that broader research that stimulates different ways of thinking about the local problem can be generative, and help districts create or adapt their own solutions to try and test on a small scale. Practitioners can then tinker with this approach based on ongoing data collection on both implementation and desired outcomes. This process invokes at least imagination, design, practical know-how, and contextual and instructional expertise, on top of extracting usable nuggets from the literature.

\section{Sample Practice-Driven Indicators}

- Educators' and other practitioners' perspectives are included throughout the process, including framing of research questions and design considerations

- Multiple perspectives inform the agenda development-stakeholders striving to think beyond business as usual with implications for practitioners, and researchers willing to sustain a long-term focus on persistent education problems

- Practice-generated questions are often multidisciplinary and implicate triangulation and intersections across knowledge and skills from the learning sciences, child and youth development, social psychology and organizational theory, change management, political science, and other disciplines

- Agenda is structured by practical, relevant constructs and content, with explicit rationale about why the questions are relevant to the field and actionable implications for stakeholders in the education system, including questions of capacity to implement and strategies for improving uptake of potential solutions

While the discussion and resulting agendas seem to reflect consistently a practice orientation, we were surprised by the lower emphasis on equity-centered questions. In hindsight, we recognize that while the practice-driven elements of our process were evident, it is less clear whether equity was centered in the discussions throughout the day. We present several reflections about the challenge of centering equity, and consider a range of improvements:

- Participants from the practitioner side tended to understand equity as ensuring teachers regularly address the learning needs of their individual students. In contrast, the research literature tends to identify persistent inequities in opportunities and perceived potential to learn among student groups and 
communities. This difference in focus between immediate, day-to-day fair practice and the need to ameliorate historical disparities meant participants had tacit and unshared understandings of "equity" throughout the session. Providing more explicit definitions, level-setting, and a deeper and more usable framing around equity early in the day would give participants common references. Specifically, extending the convening to allow time for an upfront activity that would tap into the intersection of research, practice, and equity, and provide common vocabulary, could better prime the discussion for the rest of the convening.

- Many of the convening participants' primary roles, leadership, and expertise related to adolescent literacy or CT topic content, rather than diversity, equity, and inclusion. Increasing the representation of equity researchers and district staff who lead local equity and social justice efforts at the convening might help to keep these considerations centered in the discussion.

- The research questions that participants raised were conditioned by the nature of the specific challenges districts presented. Being practice-driven meant that we honored the way the district representatives characterized their challenges. However, we could have facilitated additional challenge framing with a greater and more explicit emphasis on equity. Moving beyond many districts' focus on equality and providing access to "all students," we could help district teams incorporate an equity lens by identifying target populations (e.g., Black students or English learners) to support, and inequitable outcomes that indicate the system is not adequately serving the target populations.

- It was difficult for facilitators to be prepared to push participants on equity in the moment, while also managing discussion coherence, inclusion, and session timelines. An improvement would be to designate a facilitator whose role is to track equity in the discussion, with prepared prompts or questions to introduce into the discussion when appropriate. Such prompts could acknowledge and question common fears that arise in conversations around educational equity-for example, the local political environment surrounding equality and equity distinctions, perceptions of "special treatment," and perceived risks to maintaining rigor, all of which participants cited during discussions.

Articulating equity-centered questions relies on both lens and skill to make it a consistent practice. For example, We All Count points out that often, researchers implicitly frame research questions they intend to be equity-focused as the population of concern that needs to change, rather than the system perpetuating inequity that has to change. Beyond identifying specific populations of focus, questions need to investigate the structures, processes, and norms that perpetuate systemic inequities in educational outcomes. This lens then needs to be married with the skill of formulating questions in a researchable way to generate equity-centered research questions. A longer, iterative approach and changes in the upfront scaffolding for equity might yield additional or revised questions that center equity and reflect equity as an intentional, driving force rather than an add-on or a layer, however well-meaning. 


\section{Sample Equity-Centered Indicators}

- Marginalized groups' voices and participation are present and evident from the start of shaping the R\&D agenda

- Equity is not a separate consideration but integral to the problems addressed in the agenda (e.g., into curriculum, new initiatives); the R\&D agenda aims to solve specific equity challenges faced by marginalized populations

- Equity-informed vocabulary frame questions about how the system includes and excludes specific populations 


\section{Conclusion}

The Equity in the Driver's Seat convening was a one-day experiment, both to try out a process of integrating practitioners' and researchers' perspectives and to examine the nature of R\&D agendas that might arise from this integration grounded in two specific content areas, adolescent literacy and computational thinking. The convening yielded useful protocols for centering practice in generating R\&D questions and underscored the effort and intentionality required to maintain a focus on equity throughout the conversation, despite comprehensive protocols and preparation. The resulting R\&D agendas illustrate the specificity of questions that address practitioners' concerns-in some cases, so context-dependent that broader research can only apply by extrapolation and triangulation across multiple bodies of research. In these cases, smaller pilots and trials based on local expertise may yield more usable knowledge than formal large-scale research studies.

Equity in the Driver's Seat was the beginning. We continue to learn about how to spotlight equity in identifying questions to research, creating development criteria, defining outcomes that matter to marginalized communities, and understanding implementation factors that shape inclusion. Through upcoming forums, we will expand the one-day convening to a collaborative multiple-session experience for education leaders, researchers, policymakers, and community members, using revised protocols and co-designed methods.

The principles of practice-driven, equity-centered R\&D agendas build on an Inclusive Innovation model Digital Promise proposed and is currently refining through partnerships with school districts and communities. We are committed to prioritizing the role of on-the-ground voices who bring context expertise-including teachers, students, and families - within education R\&D processes, and correcting for the historical exclusion of those whom education innovations are supposed to benefit. Additionally, we will facilitate capacity-building for researchers, developers, and communities interested in applying the approach in their respective settings, and will continue to learn and iterate on the model in order to keep equity at the center. 


\section{Acknowledgements}

We are grateful to members of the League of Innovative Schools district teams and other attendees who contributed their perspectives at the convening, and to many colleagues who generously provided input and thought partnership to this project.

\section{District Teams:}

Fox Chapel Area School District (adolescent literacy): Megan Collett and Dana Simile Indian Prairie School District (adolescent literacy): Tarah Fowler, Kathy Pease, and Jessica Walsh

Iowa City Community School District (computational thinking): Dominic Audia, Andrew Fenstermaker, and Adam Kurth

Talladega County Schools (computational thinking): Holley Burford, Brooke Morgan, and Emily Nestor

\section{Additional Convening Attendees:}

Linda Friedrich, Brian Giovanni, Jonathan Hoffman, Beatriz Orozco, Haydee Rodriguez, Rafi Santo, Allison Scott, Kennan Scott (keynote), Jessica Stovall (keynote), and Emily Thomforde

\section{Digital Promise Staff Contributors:}

Mahsa Bakhshaei, Quinn Burke, Carly Chillmon, Corinne Colgan, Tabatha Mason, Kelly Mills, Alison Shell, Kim Smith, Medha Tare, and Josh Weisgrau

Research aWork 2019-2020 Advisory Board:

Paula Arce-Trigatti, Angela DeBarger, Julie Riordan, Ash Vasudeva, and Bari Walsh

\section{Suggested Citation}

Liberman, B. \& Young, V. (2020). Equity in the driver's seat: A practice-driven, equity-centered approach for setting R\&D agendas in education. San Mateo, CA: Digital Promise. 